\title{
NAGOYA
}

MATHEMATICAL JOURNAL

\section{VOL. 39}

August, 1970

PUBLISHED BY

MATHEMATICAL INSTITUTE, FACULTY OF SCIENCE NAGOYA UNIVERSITY 
All communications relating to this publication should be addressed to the Editors of the Nagoya Mathematical Journal, Mathematical Institute, Nagoya University, Chikusa-ku, Nagoya, Japan. 


\title{
NAGOYA
}

\section{MATHEMATICAL JOURNAL}

\section{VOL. 39}

\author{
August, 1970
}

Edited by

Takeyuki Hida, Noboru Ito, Tomio Kubota, Sigekatu Kuroda, Hideyuki Matsumura, Hisasi Morikawa, Akihiko Morimoto, Kryoshi Noshiro, Katuzi Ono, Yoshihiro Shikata, Kôsaku Yoshida

PUBLISHED BY

MATHEMATICAL INSTITUTE, FACULTY OF SCIENCE NAGOYA UNIVERSITY 


\section{GONTENTS}

The Centers of Semi-Simple Algebras Over a Commutative Ring, II. S. Endo AND Y. Watanabe

Sample Properties of Weakly Stationary Processes.

................................ T. Kawata and I. Kubo 7

Note on the Characters of Solvable Groups. .................. ITo 23

On Holomorphic Families of Holomorphic Maps. ........D. ORTH 29

A Characterization of the Finite Simple Groups $P S p(4, q), G_{2}(q)$, $D_{4}^{2}(q)$, II. ..................................... Fong 39

Some Homogeneous Einstein Manifolds. Arthur A. Sagle

On the Growth of Solutions of Algebraic Differential Equations Whose Coefficients are Arbitrary Entire Functions.

S. BANK 107

Propriétés des Solutions Faibles Non Négatives de L'équation Parabolique. J. Chabrowski

On a Problem of Doob Concerning Multiply Superharmonic Functions. K. Gowrisankaran

The Behaviour of Meromorphic Functions with a Set of Singularities of the Class $N_{B}^{0}$. .A. Sagawa 133

Integral Normal Bases in Galois Extensions of Local Fields. S. ULIOM

Normal Light Interior Functions Defined in the Unit Disk.

J.H. Mathews 149

Evans-Kuramochi Exhaustion Functions on Non-Algebroid Riemann Surfaces. .J.H. SKeAth

Elementary Quotients of Abelian Groups, and Singular Homology on Manifolds.

Marston Morse and Stewart Scott Cairns

Correction to "Green's Functions for Generalized Schroedinger Equations" in Nagoya Mathematical Journal, Vol. 35. ... John A. BeEkMAN 